recognising and management of delirium and agitation after the educational intervention

\section{P-96 DIAGNOSIS AND ASSESSMENT OF DELIRIUM IN HOSPICE INPATIENTS}

Holly McGuigan, Gillian Scott, Ruth Isherwood. Strathcarron Hospice, Denny, UK

10.1136/bmjspcare-2017-hospice.122

Background Delirium may be reversible in up to $50 \%$ of patients with advanced cancer and the majority of patients who recover from delirium recall it as highly distressing.

Aims To retrospectively identify episodes of delirium in hospice inpatients and explore practice around consideration and management of potentially reversible causes.

Methods A retrospective review of all patients who were inpatients in the hospice during a two-week period was performed by searching electronic records for use of descriptors agreed by a group of healthcare professionals to be reasonable proxy terms for delirium. For each delirium episode the following were considered:

- Whether a diagnosis of delirium was made

- Whether the most likely cause was identified and addressed where appropriate.

Results 28 patients were included in the project with 38 episodes of delirium identified in total. A diagnosis of 'delirium' was made in 13 of these 38 episodes. In 23 of the 38 episodes a most likely cause was identified. An attempt was made to address the documented most likely cause in 16/23 cases. In seven this was either not possible or thought to be inappropriate due to the patient's condition.

Discussion Proxy descriptors were used to identify delirium episodes. The use of proxy descriptors is likely to underestimate hypoactive delirium which is thought to represent the majority of delirium seen in hospice populations. It was more difficult to assess from descriptors of hypoactive delirium e.g., drowsy or sleepy if symptoms are related to delirium or to disease progression. A quality improvement project has been established to improve recognition and assessment of delirium. A validated screening tool has been introduced. Education has focused on prompt identification and active consideration of possible causes of delirium, and whether it is possible and appropriate to reverse these.

\section{P-97 AN AUDIT OF THE ASSESSMENT OF DELIRIUM IN HOSPICE ADMISSIONS THROUGH THE INTRODUCTION OF THE 4AT}

Elizabeth Fleming. Gateshead NHS, Gateshead, UK

\subsection{6/bmjspcare-2017-hospice.123}

Background Delirium is a significant problem in the palliative care setting, with incidence of delirium ranging from 5\%-45\% of inpatients (Hosie et al., 2013). In Scotland there has been development of a new management pathway for delirium by Healthcare Improvement Scotland and the Scottish Delirium Association (2016). This recommends the use of the 4AT to help diagnose delirium.

Aims This is an audit to review the practice of screening for delirium in the hospice setting. The aim of the audit is to ensure delirium assessment in the hospice is in line with current guideline. The standard is that $100 \%$ of patients over the age of 65 should have a documented cognitive screen on admission.

Methods Notes from patients admitted from 19 June to 19 November 2015 were retrospectively reviewed to assess for formal documentation of delirium screening and also the incidence of delirium in this population. Patients over the age of 65 were included and patients that were admitted for care in the last days of life were excluded from the data. Stage two involved the introduction of the 4AT in the above inpatient population. The use of the 4AT was then re-audited in patients who were admitted to the hospice in the following six months.

Results Prior to the intervention only three out of 38 patients had formal assessment of delirium on admission. Post intervention 26/29 patients had formal assessment with the use of the 4AT. $58 \%$ patients who were admitted over this year period and fitted the inclusion criteria developed a delirium during their stay.

Conclusion This audit has shown that education and introduction of simple routine assessment can increase the recognition of delirium in a palliative care setting, potentially improving levels of care for patients.

\section{P-98 PALLIATIVE CARE AND DEMENTIA COLLABORATION PROJECT}

Nicola Sexton, Karen Chumbley. St Helena Hospice, Colchester, UK

10.1136/bmjspcare-2017-hospice. 124

Background There are unmet palliative care needs in dementia (Van der Steen et al., 2013; Dempsey et al., 2015). The number of people dying with dementia is rapidly increasing (Etkind et al., 2017). Choice in end of life care is a national priority (National Palliative \& End of Life Care Partnership, 2015; Department of Health, 2015). NICE Guidance recommends a palliative care approach from point of diagnosis and equitable access to services based on need (NICE clinical guideline reviewed, 2016; Dixon et al., 2015). Electronic care registers (My Care Choices Register in North East Essex) enables recording and sharing of care preferences (Wee, 2015; Department of Health, 2015). Hospices have a pivotal role in sharing palliative care expertise and enabling other teams to incorporate palliative care principles and practices into their work (Calanzani et al., 2013; Hospice UK., 2015). Palliative care teams also need to develop skills in dementia (Van der Steen et al., 2013; Hospice UK., 2015). We therefore developed the Palliative Care and Dementia Project in September 2016.

Aims 1) Develop collaborative working between Palliative Care and Mental Health.

2) Identify unmet palliative care needs in advanced dementia.

3) Increase number of referrals for Dementia patients.

4) Establish use of My Care Choices Register.

Methods Senior Palliative Doctor providing weekly direct support to two Acute Dementia wards over six months. Baseline and subsequent audit of My Care Choices Register registration and hospice referrals. Use of SPICT (10) - Supportive and 
Palliative Indicators Tool - to aid identification of palliative care needs. 10 hours teaching.

Results

- Hospice referrals for dementia patients increased: 2015/16 $76 ; 2016 / 17153$.

- Referrals from mental health increased: 2016/17 one referral; 2016/17 six referrals.

- Baseline audit of 24 inpatients demonstrated two were already on My Care Choices Register and mental health ward staff were unaware of this

- By May 2017 My Care Choices Register checked for every admission, seven referrals, three received end of life care on the ward.

- Issues - pain, agitation, nutrition/hydration, ethical dilemmas, falls, infection, advance care planning, carer burden.

- Other outcomes - positive educational feedback, reciprocal job shadowing (nurses), Namaste training (Stacpoole et al., 2015).

Conclusion This project innovatively enhanced collaborative working, increased referrals and established the My Care Choices Register in this setting. Dementia inpatient teams were enabled to improve skills and confidence in providing palliative care. Symptom management was addressed and hospital admissions were avoided for dying patients. Reciprocal educational needs were highlighted. Further work is being undertaken to consolidate this work, identify further dementia/ palliative care educational needs and build on the collaboration.

\section{P-99 DEMENTIA CARE IS EVERYONE'S BUSINESS}

${ }^{1}$ Helen Reeves, ${ }^{2}$ Angela Causton, ${ }^{3}$ Michael Hurt. ${ }^{1}$ St Giles Hospice, Whittington, UK; ${ }^{2}$ Pathways 4 Life, Walsall, UK; ${ }^{3}$ NHS Walsall Clinical Commissioning Group, Walsall, UK

\subsection{6/bmjspcare-2017-hospice.125}

Background Studies have found that people with dementia are often admitted to hospital unnecessarily from care homes by staff who have not received enough support to help them understand dementia and how to support them at the end of life.

Aims The presentation will cover how working collaboratively has resulted in a change across the culture of Walsall healthcare resulting in improved outcomes for patients with dementia. It will provide practical solutions to commonly found problems when looking at service improvements and provide an honest account of setting up a collaborative partnership between organisations and the challenges we encountered and the ways we overcame them.

Method Support is provided by a range of methods including observations, support sessions, forums and steering groups. Homes can access as much or as little as they want and have ongoing contact to ensure their needs are met. Collaboratively work with other services for more joined up working.

Results As a direct result of the service we now have a community dementia steering group that brings together a range of professionals including care homes, ambulance service, hospital, hospice, community professionals, commissioners to name a few. Here we focus on how we can all work collaboratively to improve care for dementia patients. The service is now recurrently funded and this in part is due to the service having a direct impact on the decreasing the number of patients with dementia being admitted to the acute sector.

Conclusion There is no quick fix to improving cross boundary and sector working, however, as proven by this service by acknowledging that we all have a part to play and that we can all positively influence patients care change does not have to cost anything other than time and the dedication of the staff involved.

\section{P-100 SYSTEMATIC REVIEW OF FACILITATORS AND CHALLENGES TO DYING AT HOME WITH DEMENTIA}

${ }^{1}$ Caroline Mogan, ${ }^{1}$ Mari Lloyd-Williams, ${ }^{2}$ Karen Harrison Dening, ${ }^{1}$ Chris Dowrick. ${ }^{1}$ Academic Palliative and Supportive Care Studies Group, Institute of Psychology Health and Society, University of Liverpool, UK; ${ }^{2}$ Dementia UK, UK

\subsection{6/bmjspcare-2017-hospice. 126}

Background Place of death is important in end of life care and it is reported that given the right support, most people would choose to die at home. A very small minority of people with dementia die at home and knowledge gaps remain on how best to support end of life care at home for people with dementia.

Aim This systematic review synthesised qualitative and quantitative studies to explore the challenges and facilitators of providing end of life care at home for people with dementia.

Methods A narrative approach was adopted to provide a comprehensive synthesis of previously published literature in the area. A systematic literature search was conducted across six electronic databases (AMED, BNI, CINAHL, EMBASE, MEDLINE, and PsychInfo) and reference lists of key journals were searched up to November 2016. Included studies were appraised for quality and data thematically synthesised.

Results Searches returned 1949 unique titles, of which seven studies met all eligibility criteria (four quantitative, three qualitative). All studies were rated for quality and six key themes identified - four facilitators and two challenges. Facilitators included 'support from health care professionals', 'informal caregiver resilience', 'medications and symptom management' and 'appropriate equipment and home adaptations'. Challenges included 'worsening of physical or mental health' and 'issues with formal services'.

Conclusions People with dementia may not always require specialist palliative care at the end of life and many clinicians, services and charities support people with dementia to die well at home. Informal caregivers also provide a significant amount of this care to people. Further research is required to establish how clinicians, statutory services, voluntary agencies and volunteers can support families to allow a greater number of people with dementia to die at home and to determine what services are required to facilitate home death for people with dementia.

\section{P-101 DEVELOPING A COMMUNITY DEMENTIA COMPANION SERVICE - EXTENDING VOLUNTEER LED HOSPICE SERVICES}

Claire Halsey, Lyn Deakin, Dawn Lawton. East Cheshire Hospice, Macclesfield, UK

10.1136/bmispcare-2017-hospice. 127 